\title{
MmWave Codebook Selection in Rapidly-Varying Channels via Multinomial Thompson Sampling
}

\author{
Yi Zhang \\ The University of Texas at Austin \\ Austin, TX, USA \\ yi.zhang.cn@utexas.edu \\ Sanjay Shakkottai \\ The University of Texas at Austin \\ Austin, TX, USA \\ sanjay.shakkottai@utexas.edu
}

\author{
Soumya Basu \\ Google, LLC \\ Mountain View, CA, USA \\ basusoumya@google.com \\ Robert W. Heath Jr. \\ North Carolina State University \\ Raleigh, NC, USA \\ rwheathjr@ncsu.edu
}

\begin{abstract}
Millimeter-wave (mmWave) communications, using directional beams, is a key enabler for high-throughput mobile ad hoc networks. These directional beams are organized into multiple codebooks according to beam resolution, with each codebook consisting of a set of equal-width beams that cover the whole angular space. The codebook with narrow beams delivers high throughput, at the expense of scanning time. Therefore overall throughput maximization is achieved by selecting a mmWave codebook that balances between beamwidth (beamforming gain) and beam alignment overhead. Further, these codebooks have some potential natural structures such as the non-decreasing instantaneous rate or the unimodal throughput as one traverses from the codebook with wide beams to the one with narrow beams. We study the codebook selection problem through a multi-armed bandit (MAB) formulation in mmWave networks with rapidly-varying channels. We develop multiple novel Thompson Sampling-based algorithms for our setting given different codebook structures with theoretical guarantees on regret. We further collect real-world $(60 \mathrm{GHz})$ measurements with 12-antenna phased arrays, and show the performance benefits of our approaches in an IEEE 802.11ad/ay emulation setting.
\end{abstract}

\section{CCS CONCEPTS}

- Networks $\rightarrow$ Mobile networks; • Computing methodologies $\rightarrow$ Machine learning algorithms; • Mathematics of computing $\rightarrow$ Bayesian computation.

\section{KEYWORDS}

millimeter-wave, codebook optimization, rapidly-varying channel, multi-armed bandit, Thompson sampling, experimental measurements

Permission to make digital or hard copies of all or part of this work for personal or classroom use is granted without fee provided that copies are not made or distributed for profit or commercial advantage and that copies bear this notice and the full citation on the first page. Copyrights for components of this work owned by others than the author(s) must be honored. Abstracting with credit is permitted. To copy otherwise, or republish, to post on servers or to redistribute to lists, requires prior specific permission and/or a fee. Request permissions from permissions@acm.org.

MobiHoc '21, July 26-29, 2021, Shanghai, China

(c) 2021 Copyright held by the owner/author(s). Publication rights licensed to ACM ACM ISBN 978-1-4503-8558-9/21/07 ..\$15.00

https://doi.org/10.1145/3466772.3467044

\section{ACM Reference Format:}

Yi Zhang, Soumya Basu, Sanjay Shakkottai, and Robert W. Heath Jr.. 2021. MmWave Codebook Selection in Rapidly-Varying Channels via Multinomial Thompson Sampling. In The Twenty-second International Symposium on Theory, Algorithmic Foundations, and Protocol Design for Mobile Networks and Mobile Computing (MobiHoc '21), fuly 26-29, 2021, Shanghai, China. ACM, New York, NY, USA, 10 pages. https://doi.org/10.1145/3466772.3467044

\section{INTRODUCTION}

Large antenna arrays are key to the success of millimeter-wave (mmWave) networks because of their high directional gain. However, to get the benefits of this directionality, transmitters (TX) and receivers $(\mathrm{RX})$ need to align their respective beams to maximize throughput. Each radio has a codebook - a collection of beams with a predefined beam resolution (indicated by beamwidth), and covering the whole angular space (see Figure 1) - the radios exhaustively sweep over the beams in a codebook to establish the optimal beam-pair link [29]. Such sweep-based techniques have been incorporated into standards such as IEEE 802.11ad/ay [4] and $5 \mathrm{G} \mathrm{NR}$ [5], because of robustness and good coverage [28].

While a codebook consisting of beams with a narrow beamwidth is beneficial as these beams provide higher beamforming gain (and thus a higher signal-to-noise ratio (SNR)), it comes at a price. Such a codebook correspondingly contains a large number of beams to cover angular space, with the time taken to sweep over them being linear in the number of beams [17]. Indeed with emerging standards such as IEEE 802.11ay, the number of beams can scale to as much as 2048 [4, 26]. Furthermore, a beam-pair link needs to be frequently re-established in mobile and rapidly varying channel settings (see [9]), thus resulting in significant overheads.

To resolve this tension between high throughput and large sweep times, a promising and practical solution is to have multiple codebooks of different beam resolutions (each codebook spanning the whole angular space, see Figure 1 and Remark 1), and choose a specific codebook in a scenario-specific manner. Depending on the device location and frequency of link realignment (which is driven by scenario-specific device location/mobility, and channel variability), the radio might choose to use a codebook of wide beams (low beamforming gain but fast sweep, beneficial to devices that either require frequent realignment or can tolerate low beamforming gain due to their central location), or at the other extreme, a codebook of narrow beams (high beamforming gain but slow sweep, beneficial 
to devices requiring infrequent realignment or located far-away from the base station). Indeed the experiments in [37] have shown that the optimal beam resolution is scenario-specific, and unsuitable choices could severely degrade the overall throughput. This intuition has propagated into standards, where a family of codebooks has been first standardized in IEEE 802.15.3c millimeter-wave WPANs [1] and further proposed in the ongoing standardization of IEEE 802.11ay by [26].

In this paper, we focus on the codebook selection problem given a set of mmWave codebooks ranging from low to high beam resolution (see Figure 1). Our goal is to learn the optimal codebook by dynamically exploring the trade-off between the high instantaneous throughput provided by the codebook of narrow beams and the low overhead associated with the codebook of wide beams. We exploit online learning techniques to design codebook selection algorithms for rapidly-varying mmWave networks. The major contributions are summarized below:

(1) Algorithm Design: Using a multi-armed bandit (MAB) framework, we propose multiple novel Thompson Sampling (TS)-based bandit algorithms using Dirichlet priors for the codebook selection problem. In particular, we first propose a generic TS algorithm without requiring any structure among codebooks. Second, we propose a constrained TS algorithm that exploits the known general structure among codebooks to further improve the system performance. Most importantly, we propose a Unimodal TS (UTS) algorithm to deal with a well-observed natural structure among a family of codebooks ranging from low to high resolution - the effective throughputs of codebooks often have a unimodal property.

(2) Theoretical and Empirical Results: We provide theoretical guarantees for the proposed algorithms by deriving upper bounds for their regrets (expected loss in cumulative throughput) with respect to a genie algorithm that always uses the optimal codebook. In particular, our proofs provide the theoretical guarantee for the UTS with Dirichlet priors, which is an important missing part of the state-of-the-art TS algorithms. Next, we collect real-world channel measurements at $60 \mathrm{GHz}$ with two 12-antenna phased arrays, and use them to validate the proposed algorithms by emulating an IEEE 802.11ad system. Our results show that the proposed TS-based algorithms are superior to state-of-the-art bandit algorithms.

\section{SYSTEM MODEL}

We consider a slot-based mobile ad hoc mmWave system, in which a TX establishes the wireless link with an RX by doing the codebookbased beam scanning. Specifically, a codebook is a set of directional beams of the same beam resolution (indicated by beamwidth) that covers the whole angular space. There are multiple codebooks available at the TX while the RX only has one fixed codebook (antenna array size and power consumption are generally limited at the RX, i.e. mobile devices). Different codebooks have directional beams of different beamwidth, which helps balancing high beamforming gain (by delivering high SNR using narrow beams) and low training overhead (by avoiding mass sweeps using wide beams). See Figure 1 for a pictorial representation of the set of codebooks.

In mmWave systems, each communication time slot includes a beam alignment phase and a data transmission phase. The evolution of a time slot is described as follows. At the beam alignment

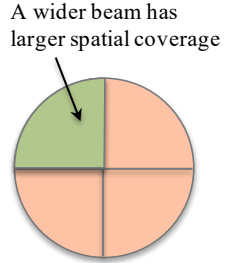

1-th codebook
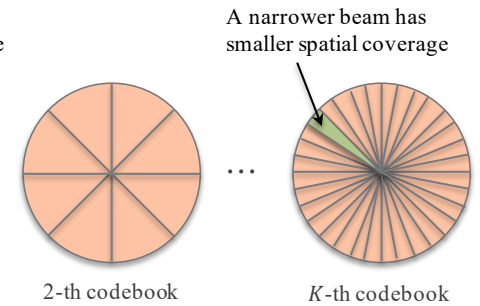

Figure 1: Example codebooks of directional beams

phase, the TX selects one of the available codebooks to perform the beam alignment with the RX by testing all the beams in this codebook. At the end of this phase, the index of the beam with the highest received signal strength (RSS) will be sent back to the TX. Subsequently, the TX will use this best beam to transmit data for the remaining time resources in this slot, which is referred to as a data transmission phase. In particular, the TX will transmit the data with the highest supportable modulation and coding scheme (MCS), which is obtained by referring to a predefined RSS-MCS table. This is a typical mmWave system and the adopted beam alignment process is similar to the sector level sweep (SLS) used in IEEE $802.11 \mathrm{ad} / \mathrm{ay}[3,4]$ and 5G NR [5]. Our objective is to identify the optimal codebook that maximizes the expected system throughput.

The codebook generation is out of the scope of this work. A simple way to generate multiple codebooks of different beamwidths, shown in Figure 1, is to exploit antenna on/off techniques [40, 42], which is also used in our experimental evaluation.

REMARK 1. Compared to gathering all the beams of different resolutions into a giant codebook, organizing the beams into multiple codebooks by their width has the following practical advantages: (1) It facilitates the beam management in the context that the size of the mmWave antenna array is scaling up [43]. (2) It enables the codebook optimization in a scenario-specific manner (see experimental results in [37]), leading to greatly improved performance. (3) From the perspective of practical implementation, using one codebook of equal-width beams for a single link establishment can avoid numerous antenna on/off operations (required by changing beamwidth [40]), which could reduce the operation overhead and simplify the antenna hardware designs. As mentioned earlier, standard bodies are recognizing the benefits of a family of codebooks, e.g. IEEE 802.15.3c millimeter-wave WPANs [1] and proposals in IEEE 802.11ay by [26].

\section{PROBLEM STATEMENT}

In this section, we mathematically characterize the beam alignment and the data transmission phases described in Section 2. We study the codebook selection problem through a multi-armed bandit (MAB) framework. At each time slot, one of $K$ possible codebooks (aka actions) is chosen by the learning algorithm (aka player), and the corresponding effective data rate (aka reward) is observed. By learning the choice of the best codebook, the goal is to minimize the cumulative loss with respect to an omniscient genie [8].

\subsection{RSS-MCS table}

As mentioned in Section 2, there exists a predefined RSS-MCS table used by the TX to decide which is the highest supportable MCS 
given the best RSS feedback by the RX. We suppose this RSS-MCS table has $(M+1)$ levels of MCS. The data rate associated with MCS $m$ is the $m$-th element of a rate vector $\tilde{\mathbf{r}}=\left[\tilde{r}_{0}, \tilde{r}_{1}, \ldots, \tilde{r}_{M}\right]^{\mathrm{T}}$, where $\tilde{r}_{0}<\tilde{r}_{1}<\ldots<\tilde{r}_{M}$, and the minimum required RSS for supporting MCS $m$ is denoted as $\operatorname{rss}_{m}$, which yields a RSS vector rss $=\left[\operatorname{rss}_{0}, \mathrm{rss}_{1}, \ldots, \mathrm{rss}_{M}\right]^{\mathrm{T}}$. In particular, MCS 0 represents the data rate of $0\left(\tilde{r}_{0}=0\right.$ and $\left.\operatorname{rss}_{0}=-\mathrm{inf}\right)$, namely that the RSS is too low to support any data transmission (failed link connection). Without loss of generality, we define a normalized rate vector by dividing $\tilde{\mathbf{r}}$ by $\tilde{r}_{M}$, which is denoted as $\mathbf{r}=\left[r_{0}, r_{1}, \ldots, r_{M}\right]^{\mathrm{T}}$, where $r_{m}=\frac{\tilde{r}_{m}}{\tilde{r}_{M}}$. Thus, $r_{m}$ is bounded by $[0,1]$ and we will use this normalized rate vector $\mathbf{r}$ in the following. We denote $[K]^{+} \triangleq\{1,2, \ldots, K\}$, $[K] \triangleq\{0,1, \ldots, K\}$ and $\mathbf{1}\{\cdot\}$ as the indicator function for later use.

\subsection{Channel distribution and evolution of a time slot}

We consider a discrete-time setting, where $t=1,2, \ldots, T$ is a finite time horizon and each time step represents a communication time slot. We denote $K$ as the number of codebooks at the TX and $S_{k}$ as the number of beams in the $k$-th codebook. We denote the random mmWave channel at time slot $t$ as $h(t)$ following a discrete state channel distribution $\mathcal{H}$ over some (possibly) continuous state-space. As the channels are rapidly varying in mmWave MANETs, we suppose that the channel state realizations of different time slots are independent of each other [20].

In each time slot, at the beam alignment phase, the TX chooses a codebook $k \in[K]^{+}$and sequentially tests each beam in this codebook (beam alignment for the specified codebook). Denoting by $\operatorname{rss}(\mathrm{t}, \mathrm{k})$ the maximum RSS obtained by sweeping over all the beams in the $k$-th codebook, we then have

$$
\operatorname{rss}(t, k)=\max _{j \in\left[S_{k}\right]^{+}} f(h(t), k, j),
$$

where $f$ is an unknown function that reflects the overall physical layer impact on the received signals, which includes channel gain, sidelobe effects, RF impairments, beam pattern imperfection, thermal noise, etc.

Given the maximum RSS, the TX uses a predefined RSS-MCS table to determine the highest supportable MCS for the data transmission phase, which can be mathematically expressed as

$$
r(t)=\max _{m \in[M]} 1\left\{\operatorname{rss}(t, I(t)) \geq \operatorname{rss}_{m}\right\} r_{m},
$$

where $I(t)$ denotes the index of codebook selected at the $t$-th time slot and $r(t)$ is the determined data rate, which is termed as instantaneous data rate. As a result, we can see that given a selected codebook $I(t) \in[K]^{+}$by a certain policy, the instantaneous data rate $r(t)$ follows a one-trial multinomial distribution with the support $\left\{r_{0}, r_{1}, \ldots, r_{M}\right\}$ and the parameter $\mathbf{p}_{k}=\left[p_{0, k}, p_{1, k}, \ldots, p_{M, k}\right]^{\mathrm{T}}$, where $p_{m, k}=\mathbf{P}\left\{r(t)=r_{m} \mid I(t)=k\right\}, m \in[M]$ and $k \in[K]^{+}$.

\subsection{Reward of codebooks and cumulative regret of the system}

We adopt a model-free framework to formulate our codebook selection problem, which directly characterizes the performance of codebooks by their multinomial distributions, i.e. parameters $\left\{\mathbf{p}_{k}\right\}_{k=1}^{K}$. This allows us to bypass the complex assumptions on the channel distribution $\mathcal{H}$ and the unknown function $f$ in (1). The performance metric of the $k$-th codebook (the mean reward of $k$-th arm) is the effective data rate of the codebook, $r_{k}^{\text {eff }}(t)$ (defined shortly). We first denote $r_{k}^{\text {ins }}(t)$ as the instantaneous data rate of codebook $k$, whose expectation can be given as $\mathbf{E}\left[r_{k}^{\text {ins }}(t)\right]=\mathbf{r}^{\mathrm{T}} \mathbf{p}_{k}$. As described before, only part of the total time slot is used for data transmission, which motivates us to define a variable, termed as effective coefficient, to present the ratio of time that is allocated for the data transmission phase, which is given as $C_{k}^{\text {eff }}=\left(T^{\text {slot }}-T_{k}^{\text {train }}\right) / T^{\text {slot }}$, where $T_{k}^{\text {train }}$ is a codebook-dependent constant representing the total beam alignment time including getting feedback and $T^{\text {slot }}$ is the fixed time slot duration.

With $C_{k}^{\text {eff }}$, we can now define the effective data rate, denoted by $r_{k}^{\text {eff }}(t)$, to represent the average data rate over the whole time slot, which is given as $r_{k}^{\text {eff }}(t)=r_{k}^{\text {ins }}(t) C_{k}^{\text {eff }}$. Note that $r_{k}^{\text {eff }}(t)$ determines the real system throughput when the $k$-th codebook is chosen. Therefore, the reward of $k$-th arm follows a multinomial distribution with the support $\left\{r_{0} C_{k}^{\text {eff }}, r_{1} C_{k}^{\text {eff }}, \ldots, r_{M} C_{k}^{\text {eff }}\right\}$ and the parameter $\left\{p_{0, k}, p_{1, k}, \ldots, p_{M, k}\right\}$, which gives its expectation $\mu_{k}$ as

$$
\mu_{k}=\mathbf{E}\left[r_{k}^{\mathrm{eff}}(t)\right]=C_{k}^{\mathrm{eff}} \mathbf{r}^{\mathrm{T}} \mathbf{p}_{k} .
$$

The optimal codebook $k^{*}=\arg _{k \in[K]^{+}} \max \mu_{k}$ is the one that provides the maximum expected effective data rate.

In this work, we consider minimizing the expected cumulative regret/loss over the $T$ slots. The expected cumulative regret of a codebook selection algorithm is defined as the difference between the total expected reward of the optimal codebook and the total expected reward obtained by the algorithm, which can be given as

$$
R(T)=\sum_{t=1}^{T} \mathbf{E}\left[r_{k^{*}}^{\mathrm{eff}}(t)\right]-\mathbf{E}\left[r_{I(t)}^{\mathrm{eff}}(t)\right]=T \mu_{k^{*}}-\sum_{t=1}^{T} \mu_{I(t)} .
$$

\subsection{Natural structure among codebooks and discussions}

In this subsection, we incorporate the physical layer structural aspects of the codebooks as model assumptions. The following Assumption 2 leverages the fact that aligned narrower beams provide higher beamforming gain, hence larger RSS as compared to their wider counterparts. Without loss of generality, we assume that the codebooks are numbered in terms of decreasing beamwidth (widest beamwidth numbered 1 ).

Assumption 2 (Nondecreasing InStANTANEOUS DATA RATE). For any two codebooks with indexes $k_{1}$ and $k_{2}$, such that $k_{1}<k_{2}$, for all time $t \geq 1, r s s\left(t, k_{1}\right) \leq r s s\left(t, k_{2}\right)$ holds.

Assumption 2 implies that a higher (non-lower) MCS can be supported by the codebook with larger index (finer beamwidth), which is mathematically given as

$$
\mathbf{r}^{\mathrm{T}} \mathbf{p}_{1} \leq \mathbf{r}^{\mathrm{T}} \mathbf{p}_{2} \leq \ldots \leq \mathbf{r}^{\mathrm{T}} \mathbf{p}_{K} .
$$

Training time for codebooks with wider beams is less, assuming training time per beam is constant, and thus we need to train fewer beams when using wider codebooks. This implies,

$$
C_{1}^{\mathrm{eff}}>C_{2}^{\mathrm{eff}}>\ldots>C_{K}^{\mathrm{eff}} .
$$


When the codebooks are efficiently designed, the following assumption is suitable for our system (see Remark 5).

Assumption 3 (Unimodal efFective DATA RATE). The expected rewards of codebooks, i.e. $\left\{\mu_{k}\right\}_{k=1}^{K}$ (with, $\mu_{k}=C_{k}^{\text {eff }} \mathbf{r}^{T} \mathbf{p}_{k}$ ) follows a unimodal pattern, i.e. there exists a unique $k^{*} \in\{1, \ldots, K\}$ such that $\mu_{k}$ is increasing with $k$ for all $k \leq k^{*}$, and $\mu_{k}$ is decreasing with $k$ for all $k \geq k^{*}$ :

$$
\mu_{1} \leq \ldots \leq \mu_{k^{*}} \geq \ldots \geq \mu_{K} .
$$

Thus, we have mathematically modeled the codebook selection problem in rapidly-varying mmWave channels as a MAB problem. In the next section, we will design efficient bandit algorithms to solve it. A few remarks on the proposed framework are further listed below for completeness.

Remark 4. We note that Assumption 2 and the equation (6) does not necessarily provide the unimodality described by (7). For example, $\left\{C_{k}^{\text {eff }}\right\}=(0.8,0.7,0.4,0.35)$ and $\left\{\mathbf{r}^{T} \mathbf{p}_{k}\right\}=(0.1,0.2,0.3,0.4)$. Similarly, Assumption 2 is not implied by Assumption 3.

REMARK 5. Assumption 3 is motivated by the fact that the system Shannon capacity is a unimodal function of beamwidth when doing a 2D beam scanning, as discussed below. We use $b_{k}$ to represent the width of beams in the $k$-th codebook. Suppose the size of the beam scanning area is $\theta$ (e.g. $\theta=360^{\circ}$ for $2 D$-scanning), then we have $T_{k}^{\text {train }}=\frac{\theta}{b_{k}} T^{\text {mer }}$, where $T^{\text {mer }}$ is the time duration for testing a single beam. Further, the beamforming gain can be roughly approximated as $\frac{C_{0}}{b_{k}}[2]$, where $C_{0}$ is a constant parameter related to the used antenna array. Thus, the Shannon capacity $r_{k}^{c a p}$ can be given as

$$
r_{k}^{\text {cap }}=B\left(1-\frac{\theta T^{\text {mer }}}{b_{k} T^{\text {slot }}}\right) \log _{2}\left(1+h \frac{C_{0} P_{T X}}{b_{k} P_{N}}\right),
$$

where $B$ is the bandwidth, $h$ is the channel effect, $P_{T X}$ is the transmit power and $P_{N}$ is the noise power. By denoting $C_{1} \triangleq \frac{\theta T^{\text {mer }}}{T^{\text {slot }}}$ and $C_{2} \triangleq$ $\frac{C_{0} P_{T X}}{P_{N}}, r_{k}^{c a p}$ is sampled from the function $r^{\text {cap }}(b)$ given as

$$
r^{c a p}(b)=B\left(1-\frac{C_{1}}{b}\right) \log _{2}\left(1+h \frac{C_{2}}{b}\right) .
$$

It can be shown that the function in (9) is unimodal with respect to $b$ [34]. The throughput (mean reward of arm), however, is an expectation of this expression over the channel effect. Our assumption essentially states that even after taking an expectation, unimodality holds. Our numerical evaluation with the 3GPP NR outdoor channel model and real-world measurements both confirm this observation. Please refer to Appendix B in the full paper of this work [41] for more discussions and simulation results on Assumption 2 and 3.

REMARK 6. We note that unimodality has been previously exploited in beam alignment [22]. Essentially, their notion of unimodality is that for a single codebook of beams, the performance of these beams has a unimodal pattern. Our notion of unimodality given in Assumption 3 is different. When we have multiple codebooks, each consisting of beams of the same resolution, the performance of these codebooks exhibit the unimodal structure. Our notion of codebook unimodality hinges on the trade-off between the increased scanning time for codebooks with a large number of narrow beams versus the increased instantaneous rate from the high directional gains.

\section{ALGORITHMS AND REGRET GUARANTEES}

In this section, we design four online learning algorithms for different structural constraints on the set of codebooks. Our objective is to design algorithms that will maximize the use of the optimal codebook. An ideal algorithmic choice for this task is Thompson Sampling (TS) which is a popular Bayesian approach to solving $\mathrm{MAB}$ problems because of its efficient implementation and excellent empirical performance $[10,25]$. The core of TS is to use the observations to dynamically update the posterior of a predefined prior distribution. The classic TS algorithms like [7, 20,21, 25] are designed for MAB problems with Bernoulli arms and thus cannot be directly applied to our problem which has weighted multinomial distribution. For our case, we adapt the recently proposed Multinomial TS (MTS) [33] which can deal with the multinomial arms. However, in our case, there are multiple differences for which appropriate adaptations are necessary.

1) First, in Algorithm 1 we design weighted MTS (WMTS) that handles the multinomial rewards $\left\{\mathbf{r}^{T} \mathbf{p}_{k}\right\}$ weighted by the coefficients $\left\{C_{k}^{\text {eff }}\right\}$. A similar weighted generalization has been done for Bernoulli rewards in [20].

2) Second, when the weights are time varying and stochastic, i.e. $\left\{C_{k}^{\text {eff }}(t)\right\}$ are i.i.d. vectors with mean $\left\{E\left[C_{k}^{\text {eff }}\right]\right\}$, we design Algorithm 2, general MTS (GMTS), which modulates the prior update with observations $\left\{C_{k}^{\text {eff }}(t)\right\}$ after codebook selection.

3) In Algorithm 1 and 2, we have not incorporated the structural assumptions, i.e. Assumption 2 and 3, into our designs. We next design Algorithm 3, constrained WMTS (CWMTS), that is based on [21] which can incorporate either Assumption 2 or 3 or both.

4) Even though CWMTS can handle general constraints, its implementation has high complexity due to the posterior sampling from a constrained set. In order to move to a more practical algorithm under Assumption 3 (unimodality of the rewards), we propose unimodal WMTS (UWMTS) in Algorithm 4. This algorithm carefully combines the techniques in [33] to handle multinomial rewards, with the leader-tracking based procedure of $[31,36]$ to present the improved regret guarantees.

In all the above settings, we provide theoretical guarantees on the upper bounds of the cumulative regrets.

\subsection{Notations}

We present the following notations for later use in this section: $\boldsymbol{\mu}=$ $\left[\mu_{1}, \ldots, \mu_{K}\right]^{\mathrm{T}}, \boldsymbol{\alpha}_{k}=\left[\alpha_{0, k}, \ldots, \alpha_{M, k}\right]^{\mathrm{T}}$ and $\mathbf{1}_{M}$ denotes a vector of $M$ ones. $\operatorname{Dir}\left(\boldsymbol{\alpha}_{k}\right)$ denotes the Dirichlet distribution with parameter vector $\boldsymbol{\alpha}_{k}$. We use Bernoulli $(p)$ to represent a Bernoulli pmf with success probability of $p$. We use KL ( $\mathbf{p}, \mathbf{g})$ to represent the KullbackLeibler divergence between two one-trial multinomial distributions parameterized by probability vector $\mathbf{p}$ and $\mathbf{g}$, i.e. two categorical distribution, and we define that $\mathcal{K}_{\text {inf }}(\mathbf{p}, \mu \mid \mathbf{s})=\inf \left\{\operatorname{KL}(\mathbf{p}, \mathbf{g}) \mid \mathbf{s}^{\mathrm{T}} \mathbf{g}>\mu\right\}$. We use scalar $a_{k}$ to represent the $k$-th element of a vector which is denoted by a bold font a, where $k$ could start with 0 or 1 , depending on the context. We denote $\mathcal{P}$ as a problem parameter set that contains all information of our codebook selection problem, i.e. $\mathcal{P}=\left\{\mathbf{r}, \mathbf{p}_{k}, C_{k}^{\text {eff }}, \forall k \in[K]^{+}\right\}$. 


\subsection{Algorithm without prior knowledge of structural properties}

In this subsection, we propose the Weighted Multinomial Thompson Sampling (WMTS) algorithm, which does not require any prior knowledge of the structure among the performance of arms. We maintain $K$ Dirichlet priors, which are conjugate priors for the multinomial reward distributions $\left\{\mathbf{p}_{k}\right\}_{k=1}^{K}$, for the $K$ arms individually. The details of WMTS is given in Algorithm 1. The term Weighted emphasizes that different effective coefficient $C_{k}^{\text {eff }}$ scales the support of each arm differently. The performance guarantee of WMTS is given by the following Theorem 7 .

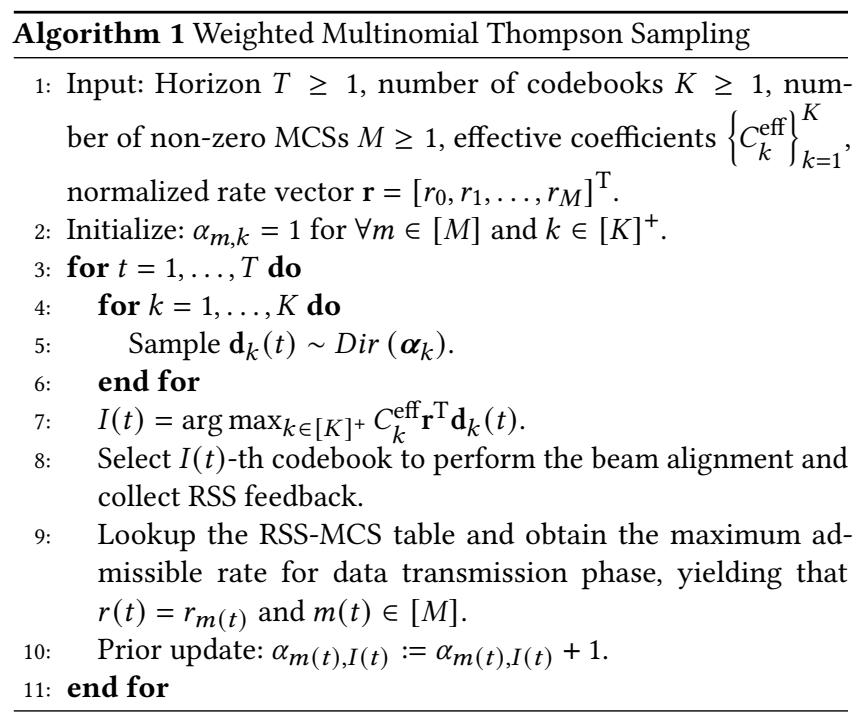

THEOREM 7. For the codebook selection problem with the access to $\left\{C_{k}^{\mathrm{eff}}\right\}_{k=1}^{K}$, WMTS has the following problem-dependent regret bound for any $\epsilon_{0}>0$ :

$$
R(T) \leq \sum_{k=1, k \neq k^{*}}^{K} \frac{\left(1+\epsilon_{0}\right)\left(\mu_{k^{*}}-\mu_{k}\right)}{\mathcal{K}_{\text {inf }}\left(\mathbf{p}_{k}, \mu_{k^{*}} \mid C_{k}^{\text {eff }} \mathbf{r}\right)} \log T+W\left(\mathcal{P}, \epsilon_{0}\right),
$$

where $W\left(\mathcal{P}, \epsilon_{0}\right)$ is a problem-dependent constant that does not depend on $T$.

Proof. The proof directly follows [33] by generalizing it to that different arms can have different supports for their respective multinomial distributions.

4.2.1 Discussion of further generalization. In this part, we briefly discuss a further generalization of Algorithm 1 when $\left\{C_{k}^{\text {eff }}\right\}_{k=1}^{K}$ are inaccessible. For the codebook-based beam training adopted in our studied system, $T_{k}^{\text {train }}$ can be easily calculated, as detailed in the evaluation section. However, if other specially designed beam alignment algorithms were used, e.g. an algorithm that terminates with a good enough beam (see the Section of related work for more examples), $T_{k}^{\text {train }}$ could be random variables whose realizations are only accessible after completing the beam alignment. This is indeed an example of generalizations of our proposed MAB framework. Motivated by this, we also derive General Multinomial Thompson Sampling (GMTS) algorithm, which is denoted as Algorithm 2 (the detailed algorithm description is omitted due to space limitation). The key step in GMTS is to randomize the reward of arm after observing the sample-path-dependent $C_{k}^{\text {eff }}(t)$, where $k=I(t)$. To be specific, we generate a Bernoulli random variable $X$ with parameter $C_{k}^{\text {eff }}(t)$, namely $X \sim \operatorname{Bernoulli}\left(C_{k}^{\text {eff }}(t)\right)$. If $X$ is zero, then we randomize the reward to be zero, i.e. $m(t)=0$.

The performance comparison between WMTS and GMTS is shown in the evaluation results. The performance guarantee of GMTS is given by the following Theorem 8 .

THEOREM 8. For a general codebook selection problem without the access to the sample-path-dependent $\left\{C_{k}^{\mathrm{eff}}(t)\right\}_{k=1}^{K}$, GMTS has the following problem-dependent regret bound for any $\epsilon_{0}>0$ :

$$
R(T) \leq \sum_{k=1, k \neq k^{*}}^{K} \frac{\left(1+\epsilon_{0}\right)\left(\tilde{\mu}_{k^{*}}-\tilde{\mu}_{k}\right)}{\mathcal{K}_{\text {inf }}\left(\tilde{\mathbf{p}}_{k}, \mu^{*} \mid \mathbf{r}\right)} \log T+W\left(\tilde{\mathcal{P}}, \epsilon_{0}\right),
$$

where $W\left(\tilde{\mathcal{P}}, \epsilon_{0}\right)$ is a problem-dependent constant that does not depend on $T, \tilde{\mathcal{P}}=\left\{\mathbf{r}, \mathbf{p}_{k}, \mathbf{E}\left[C_{k}^{\mathrm{eff}}(t)\right], \forall k \in[K]^{+}\right\}, \tilde{\boldsymbol{\mu}}=\left[\tilde{\mu}_{1}, \ldots, \tilde{\mu}_{K}\right]^{T}, \tilde{\mu}_{k}=$ $\mathbf{r}^{T} \tilde{\mathbf{p}}_{k}, \tilde{\mathbf{p}}_{m, k}=\mathbf{p}_{m, k} \mathbf{E}\left[C_{k}^{\mathrm{eff}}(t)\right]$ for $m \in[M]^{+}, \tilde{\mathbf{p}}_{0, k}=1-\sum_{m=1}^{M} \tilde{\mathbf{p}}_{m, k}$ and $k^{*}=\arg _{k \in[K]^{+}} \max \tilde{\mu}_{k}$.

Proof. With the above described randomization, all the arms follow their own multinomial distribution with a transformed parameter $\tilde{\mathbf{p}}_{k}$ but a common support $\mathbf{r}$. We can then directly apply Theorem 7 to get the regret bound given in (11).

\subsection{Algorithm using general structural properties}

In this subsection, we propose the Constrained Weighted Multinomial Thompson Sampling (CWMTS) algorithm, which leverages the prior knowledge of structural properties among codebooks summarized in Section 3.4. CWMTS is indeed an extension of WMTS, which is inspired by the constrained Bernoulli Thompson Sampling (CoTS) proposed in [21]. Its procedure is summarized as follows.

Instead of sampling $\mathbf{D}(t) \triangleq\left\{\mathbf{d}_{1}(t), \ldots, \mathbf{d}_{K}(t)\right\}$ from the product of those $K$ independent Dirichlet priors, we sample $\mathbf{D}(t)$ in the following way:

$$
\mathbf{D}(t) \propto \mathbf{1}\{\mathbf{D}(t) \in \mathbf{\Phi}\} \prod_{k=1}^{K} \operatorname{Dir}\left(\boldsymbol{\alpha}_{k}\right)\left(\mathbf{d}_{k}(t)\right),
$$

where $\Phi$ denotes the parameter space that is the set of all possible estimates of $\left\{\mathbf{p}_{k}\right\}_{k=1}^{K}$, and $\operatorname{Dir}\left(\boldsymbol{\alpha}_{k}\right)\left(\mathbf{d}_{k}(t)\right)$ is the probability density function (PDF) of $\operatorname{Dir}\left(\boldsymbol{\alpha}_{k}\right)$ for $\mathbf{d}_{k}(t)$. In particular, by omitting the time index $t$ and denoting $\mathbf{D} \triangleq\left\{\mathbf{d}_{1}, \ldots, \mathbf{d}_{k}\right\}$, under Assumption 2 , we have

$$
\Phi \triangleq\left\{\mathrm{D} \mid \mathbf{r}^{\mathrm{T}} \mathbf{d}_{1} \leq \mathbf{r}^{\mathrm{T}} \mathbf{d}_{2} \leq \ldots \leq \mathbf{r}^{\mathrm{T}} \mathbf{d}_{K}\right\},
$$

and under Assumption 3, we have

$$
\Phi \triangleq\left\{\mathbf{D} \mid C_{1}^{\text {eff }} \mathbf{r}^{\mathrm{T}} \mathbf{d}_{1} \leq \ldots \leq C_{k^{*}}^{\text {eff }} \mathbf{r}^{\mathrm{T}} \mathbf{d}_{k^{*}} \geq \ldots \geq C_{K}^{\text {eff }} \mathbf{r}^{\mathrm{T}} \mathbf{d}_{K}\right\} .
$$

Given that $I(t)$-th codebook is used and the observed reward is $r(t)=r_{m(t)}$, the prior of $\mathrm{D}(t+1)$ after Bayesian update is

$$
\begin{aligned}
& \mathbf{D}(t+1) \propto 1\{\mathbf{D}(t) \in \Phi\} \times \\
& \prod_{k=1, k \neq I(t)}^{K} \operatorname{Dir}\left(\boldsymbol{\alpha}_{k}\right)\left(\mathbf{d}_{k}\right) \times \operatorname{Dir}\left(\boldsymbol{\alpha}_{I(t)}+\mathbf{e}_{m(t)}\right)\left(\mathbf{d}_{I(t)}\right),
\end{aligned}
$$

where $\mathbf{e}_{m(t)}$ is a unit vector where the $m(t)$-th element is one. (15) shows that the update rules of priors is the same as that in the 
WMTS algorithm but we control the estimation of the distributions of arms in a more specific parameter space. We summarize CWMTS in Algorithm 3.

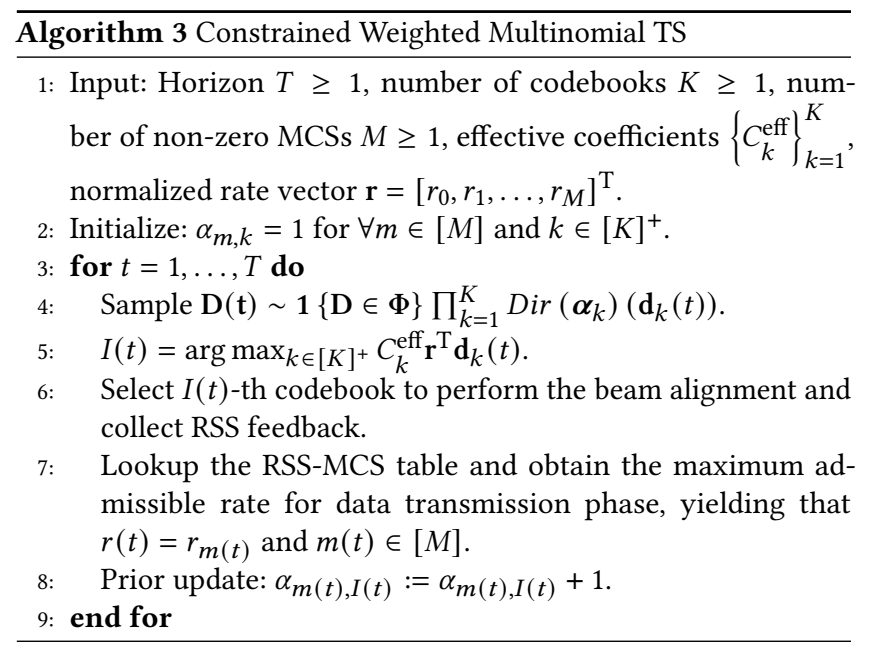

Before stating the theoretical regret bound of the CWMTS algorithm, we present the following notations. We denote $\mathcal{A}$ as the action space, namely that $\mathcal{A}=[K]^{+}$as we have $K$ codebooks. We denote $\mathcal{Y}$ as the observation space, i.e. the possible values of reward. Then we have $\mathcal{Y}=\left\{r_{m} C_{k}^{\text {eff }}, k \in[K]^{+}, m \in[M]\right\}$. We denote $\pi_{t}$ as the Dirichlet prior used in the $t$-th time slot, and denote $\pi_{0}$ is the initial prior, i.e. Dir $\left(\mathbf{1}_{M+1}\right)$, as initialized in line 2 of Algorithm 3. In addition, we make one following assumption:

Assumption 9. (Unique optimal codebook) The optimal codebook is assumed to be unique, i.e., $\mu_{k^{*}}>\mu_{k}, \forall k \neq k^{*}$.

With the above notation and Assumption 9, the following Theorem now holds.

THEOREM 10. Suppose that Assumption 9 holds, then a regret bound for the CWMTS algorithm is given as follows: For any $\epsilon, \delta \epsilon$ $(0,1)$, there exists $T^{*} \geq 0$ such that for all time horizon $T \geq T^{*}$, with probability at least $1-\delta$, CWMTS has the following problemdependent regret bound:

$$
\begin{aligned}
R(T) \leq & \left(\mu_{k^{*}}-\min _{k \in[K]^{+}} \mu_{k}\right)\left(\frac{1+\epsilon}{1-\epsilon}\right) \sum_{k=1, k \neq k^{*}}^{K} \frac{\log T}{\mathcal{K}_{\inf }\left(\mathbf{p}_{k}, \mu_{k^{*}} \mid C_{k}^{e f f} \mathbf{r}\right)} \\
& +E\left(\epsilon, \delta, \mathcal{A}, \mathcal{Y}, \Phi, \pi_{0}\right),
\end{aligned}
$$

where $E\left(\epsilon, \delta, \mathcal{A}, \mathcal{Y}, \Phi, \pi_{0}\right)$ is a problem-dependent constant that does not depend on $T$.

Proof. The proof immediately follows (with minor changes to account for multinomial instead of Bernoulli random variables) from $[19,21]$.

The above theorem shows that the regret associated with CWMTS also scales logarithmically with time as WMTS and GMTS do. Assumption 9 is made only for notational ease in the proof and it does not significantly affect the result given in Theorem 10, as pointed out in [19].
4.3.1 Discussion on the limitation of CWMTS. The straightforward way to implement CWMTS is to use rejection sampling, namely that we sample $\mathbf{D}$ from $\prod_{k=1}^{K} \operatorname{Dir}\left(\boldsymbol{\alpha}_{k}\right)$ until $\mathbf{D} \in \boldsymbol{\Phi}$. As the authors note in [21], a disadvantage of this approach is that it can be slow when the probability of getting a valid D is small. In [21], the authors proposed a heuristic Sequential Inverse Transform Sampling (SITS) approach by sampling $\mathbf{d}_{k}$ sequentially with individual constraint $\mathbf{r}^{\mathrm{T}} \mathbf{d}_{k} \leq \mathbf{r}^{\mathrm{T}} \mathbf{d}_{k+1}$. Note however that $\mathbf{d}_{k}$ are correlated with each other; thus while the heuristic SITS returns a valid sample in $\Phi$, it may not be from the correct distribution. Thereby, designing an efficient implementation of CWMTS (that results in samples from the correct distribution) is also an interesting future direction.

\subsection{Unimodal Thompson Sampling}

In this part, we present a novel algorithm exploiting the property that the effective data rates have a unimodal pattern, as stated in Assumption 3. We term it as Unimodal Weighted Multinomial Thompson Sampling (UWMTS). This is a novel combination of the Multinomial TS [33] and the Unimodal Bernoulli TS [31, 36]. The key element of this combination will be highlighted later.

To explain UWMTS, we set the following notations. We denote $N_{k}(t) \triangleq \sum_{i=1}^{t} 1\{I(i)=k\}$ as the number of times that $k$-th codebook is used up to $t$-th time slot, and the estimated expected reward of the $k$-th codebook as $\hat{\mu}_{k}(t) \triangleq \frac{\sum_{i=1}^{t} 1\{I(i)=k\} r(i) C_{k}^{\text {eff }}}{N_{k}(t)}$. In particular, we define an empirical leader to be $L(t)=\arg _{k \in[K]^{+}} \hat{\mu}_{k}(t)$, and for each arm $k$, we denote the number of times that it was a leader until time $t$ by $l_{k}(t)=\sum_{i=1}^{t} 1\{l(i)=k\}$.

Inspired by $[13,31,36]$, UWMTS focuses the search through WMTS to within a small subset around the leader, and additionally explores with the goal of detecting the optimal arm with high probability. Specifically, UWMTS chooses the arm at time $t$ by following policy:

$$
I(t)= \begin{cases}L(t) & \operatorname{Mod}\left(l_{L(t)}(t), \gamma\right)=0, \\ \text { Run WMTS in } \mathcal{N}_{L(t)}^{+}, & \text {otherwise }\end{cases}
$$

where Mod is the modulo function, $\gamma$ is the frequency that the leader is exploited, $\mathcal{N}_{k}^{+}=\mathcal{N}_{k} \cup\{k\}$ with that $\mathcal{N}_{k}$ is the set of neighboring arms of arm $k$, i.e. $\mathcal{N}_{k}=\{k-1, k+1\} \cap[K]^{+}$in our case. It is worth pointing out that there is no leader exploration when $\gamma=\infty$ and there is no theoretical guide on how to choose its value. It is empirically found by our simulation and [36] that choosing a smaller value $(2 \leq \gamma \leq K)$ results in a relatively good performance. The description of UWMTS is given in Algorithm 4.

UTS was proposed with Bernoulli arms and unimodal reward structure in [31], and it is proved to have asymptotically optimal regret in [36]. We adapt the framework in [36] and generalize the proofs therein from Bernoulli arms to multinomial arms. Such generalization, even in standard MAB (see, [33]), is known to be nontrivial as connecting the posterior of the reward (which follows Dirichlet distribution), to the observed rewards (which follows multinomial distribution) is difficult due to the absence of a closed form expression, unlike the Bernoulli case where the Beta-Binomial transform is used [6]. We leverage the tail bounds of Dirichlet distribution in [33], and derive the posterior concentration for the arms in the neighborhood of the optimal arm, which in our case 
includes two suboptimal arms and the optimal arm due to unimodality. This allows us to show each of these two suboptimal arms is played $O(\log (T))$ times in expectation, where the constant associated with the $\log (T)$ term is asymptotically optimal. Similar to [36], the other $(K-3)$ suboptimal arms are shown to be rarely played, i.e. $O(1)$ times in expectation, as the leader election method concentrates fast. Thus, we provide the first regret upper bound for UTS with multinomial arms, summarized in Theorem 11 below:

THEOREM 11. For codebook selection problem with the access to $\left\{C_{k}^{\text {eff }}\right\}_{k=1}^{K}$, under Assumption 3, UWMTS has the following problemdependent regret bound for any $\gamma \geq 2$ and any $\epsilon_{0}>0$ :

$$
R(T) \leq \sum_{k \in \mathcal{N}_{k^{*}}} \frac{\left(1+\epsilon_{0}\right)\left(\mu_{k^{*}}-\mu_{k}\right)}{\mathcal{K}_{\inf }\left(\mathbf{p}_{k}, \mu^{*} \mid C_{k}^{\text {eff }} \mathbf{r}\right)} \log T+U\left(\mathcal{P}, \epsilon_{0}, \gamma\right),
$$

where $U\left(\mathcal{P}, \epsilon_{0}, \gamma\right)$ is a constant that does not depend on $T$.

Proof. See Appendix A in the full version of this work [41].

REMARK 12. We note that UWMTS can significantly reduce the regret as the coefficient of logarithmic term is restricted to the neighborhood of the optimal arm, i.e. $\mathcal{N}_{k^{*}}$ with $\left|\mathcal{N}_{k^{*}}\right| \leq 2$. This reduces the regret from $O(K \log T)$ to $O(2 \log T)$.

\section{EVALUATION RESULTS}

In this section, we evaluate the proposed algorithms in comparison with the following state-of-the-art bandit algorithms: (1) Bernoulli Thompson Sampling (BTS) [25]: we randomize the codebook rewards to be Bernoulli random variables such that this primitive TS algorithm is applicable. (2) Weighted Bernoulli Thompson Sampling (WBTS) [20]: a modified version of BTS. (3) KL-UCB [16]: as the reward of arms are bounded by [0,1], the classic KL-UCB can be directly applied. (4) Optimal Sampling Unimodal Bandit (OSUB) [13]: OSUB is developed based on KL-UCB by further adding the leader mechanism to exploit the structural property that the rewards are unimodal. (5) Unimodal Weighted Bernoulli Thompson Sampling (UWBTS) [36]: UWBTS is a straightforward extension of WBTS by using the structural property that the rewards are unimodal.

In the following, we perform a trace-driven simulation. The simulated system adopts IEEE 802.11ad Standard, with carrier frequency of $f_{c}=60 \mathrm{GHz}$ and with a bandwidth of $B=1.76 \mathrm{GHz}[3,39]$. We incorporate the real-world channel measurements, captured at $60 \mathrm{GHz}$ and in terms of SNR, into the simulated system.

\subsection{System parameters}

In this part, we summarize the system parameters for the simulation. The duration of testing each beam $T^{\mathrm{mer}}$ is $17 \mu \mathrm{s}$ [3] and the duration per time slot $T_{\text {slot }}$ is set as $50 \mathrm{~ms}$. We adopt the RSS-MCS table provided by IEEE 802.11ad Standards for single-carrier transmission mode [3]. Accordingly, the unnormalized rate vector $\tilde{\mathbf{r}}$ is $[0,27.5$, $385,770,962.5,1155,1251.25,1540,1925,2310,2502.5,2695,3080$, $3850,4620,5005,5390,5775,6390,7507.5,8085]^{\mathrm{T}} \mathrm{Mbps}$ and the RSS vector rss is [-inf, $-78,-68,-66,-65,-64,-63,-62,-61,-60,-59$, $-57,-55,-54,-53,-51,-50,-48,-46,-44,-42]^{\mathrm{T}} \mathrm{dBm}$. By considering a noise power level of $-78 \mathrm{dBm}$, we could further compute the corresponding SNR values to get a SNR-MCS table for reference as our collected channel measurements are in terms of SNR.
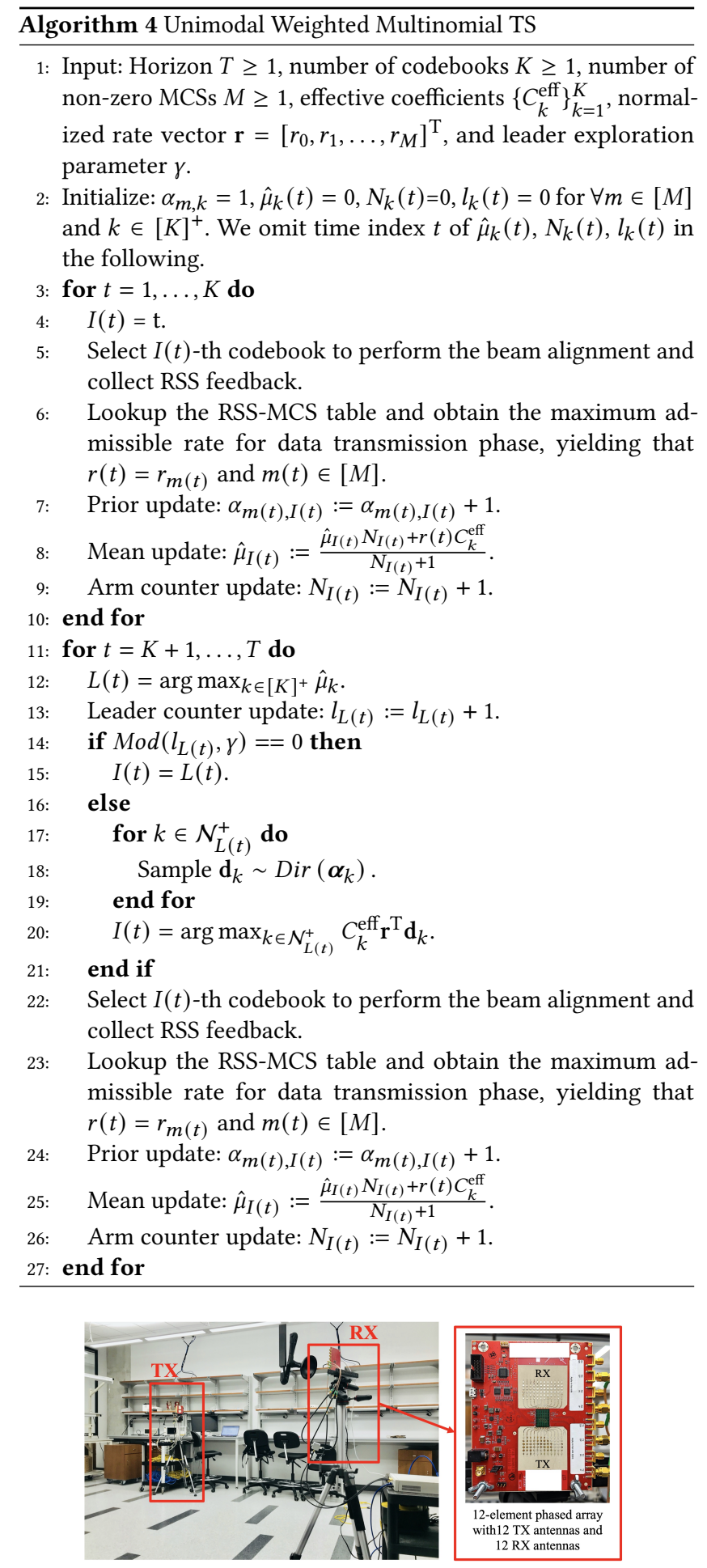

Figure 2: Experimental setup

\subsection{Real-world measurement collection}

In this part, we present our experimental setup and the collected real-world channel measurements. The testbed used for capturing 


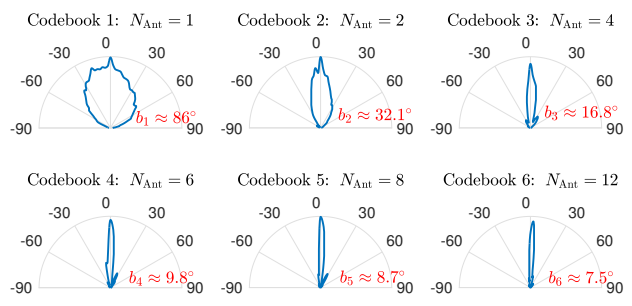

Figure 3: Example beam patterns of the 6 codebooks generated by the SiBEAM Sil6342 $60 \mathrm{GHz}$ phased arrays.

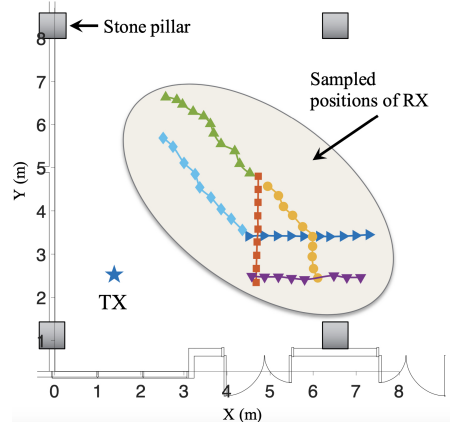

Figure 4: Sketch map of a spacious lab in which the mmWave channel measurements are taken. Different markers form different potential trajectories of $\mathrm{RX}$.

the SNR measurements consists of two 12-antenna SiBEAM Sil6342 phased arrays that up/down convert the signal to/from $60 \mathrm{GHz}$, and two N210 USRPs with a bandwidth of $5 \mathrm{MHz}$, as shown in Figure 2. By controlling the number of activated antennas $N_{\text {Ant }}$ and using phased array calibration techniques proposed by [42], we can generate directional beams of different widths. The arrays do not allow transmit power adjustment. Since our antenna array has only 12 elements, there is no major gain in having too many codebooks (as their resolutions will be too close); thus, we generate 6 representative codebooks as shown in Figure 3.

In our evaluation, we consider that $K=6$ codebooks, given in Figure 3 are available at the TX, and the RX uses the fixed Codebook 6. The size of codebook $\left\{S_{k}\right\}_{k=1}^{K}$ can be calculated with $S_{k}=\frac{360}{b_{k}}$ by considering a $2 \mathrm{D}$ beam scanning. Due to the limited bandwidth of USRP and the overhead/challenges of implementing a real-time system with user mobility, we use the testbed to measure the SNRs along certain predefined trajectories of $\mathrm{RX}$ and interpolate the values SNR with respect to the distance between TX and RX given a target velocity $(4 \mathrm{~m} / \mathrm{s})$. The sampled positions of the RX are shown in Figure 4. At each position, the SNR is measured 4 times for each codebook at the TX. Implementing a real-time system for performance evaluation would be a promising future direction but out of the scope of this work. For simplicity, we did not collect measurements for non-line-of-sight (NLOS) scenarios since we perform the beam sweeping with directional beams and the NLOS scenarios will simply result in higher path loss, which is handled by our developed MAB framework.

Based on the above setting, we further compute the values of key parameters as follows. The effective coefficients $\left(C_{1}^{\text {eff }}, \ldots, C_{K}^{\text {eff }}\right)$ is computed by $T_{k}^{\text {train }}=S_{k} S_{K} T^{\text {mer }}$ and $C_{k}^{\text {eff }}=\left(T^{\text {slot }}-T_{k}^{\text {train }}\right) / T^{\text {slot }}$, and they are $(0.9184,0.8042,0.6410,0.3962,0.3146,0.2166)$. To compute the ground truth distribution $\left\{\mathbf{p}_{k}\right\}_{k=1}^{K}$, we use the distribution statistics of the interpolated SNRs. We then draw samples from the calculated distributions during the simulation. We omit the exact values of $\left\{\mathbf{p}_{k}\right\}_{k=1}^{K}$ due to the space limitation. The expected instantaneous data rate $\left(\mathbf{r}^{\mathrm{T}} \mathbf{p}_{1}, \ldots, \mathbf{r}^{\mathrm{T}} \mathbf{p}_{K}\right)$ can be calculated as $(0.1397$, $0.2940,0.4390,0.5879,0.6626,0.7507)$. The eventual expected rewards of the $K$ codebooks $\left(\mu_{1}, \ldots, \mu_{K}\right)$ are $(0.1283,0.2364,0.2814$, $0.2329,0.2084,0.1626)$. It can be verified that the above setting satisfies both Assumption 2 and 3. We run the evaluation for $T=10000$ time slots and average the results by 200 realizations.

\subsection{Discussions on performance comparison}

In Figure 5a, we show the performance of the proposed WMTS when there is no prior knowledge of any problem structure. First, it can be seen that WMTS outperforms the state-of-the-art bandit algorithms and has a much smaller cumulative regret. Moreover, WMTS converges much faster than the other algorithms, this implies that our proposed algorithm can provide more flexibility and robustness in non-stationary environments, in which the channel distribution is time-varying. Further, we can observe that GMTS also provides a competitive performance.

In Figure 5b, we present the performance gain achieved by the CWMTS algorithm when the nondecreasing property (i.e. Assumption 2) is known to hold. As we can see, CWMTS does not provide a better regret performance than WMTS, but it converges much faster than WMTS.

In Figure 5c, we further show the performance of CWMTS and UWMTS $(\gamma=3)$ given that the unimodality property (i.e. Assumption 3) is known to hold. Some interesting observations can be drawn: (1) CWMTS outperforms OSUB $(\gamma=3)$ and UWBTS when it uses the property that the rewards have the unimodal pattern. (2) It is clear that UWMTS outperforms all the other algorithms given the unimodality, and the performance improvement is significant, which is consistent with Remark 12. (3) All the algorithms using multinomial distribution converge faster than the other algorithms.

If a random selection policy is adopted (instead of a learningbased policy), the average normalized throughput would remain at $\frac{1}{K} \sum_{k=1}^{K} \mu_{k}=0.2083$. In contrast, our online learning framework can learn the optimal codebook quickly, and the normalized throughput would be almost $\mu_{k^{*}}=0.2814$, which implies a throughput improvement by more than $35 \%$.

While the regret as a function of time provides one metric for the time taken to converge, another metric would be the number of times each codebook has been used until time $t$. Please refer to Appendix B in the full paper of this work [41] for supplementary simulation results on this. Finally, it is worth pointing out that our channel measurements are all collected under LOS condition. Appendix B in [41] also provides the evaluation results under the scenario when the NLOS condition frequently occurs.

\section{RELATED WORK}

(1) Model-driven beamwidth optimization: One of the most related lines of work is beamwidth optimization. In [34], the authors 


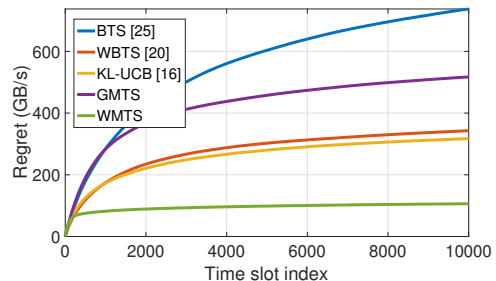

(a)

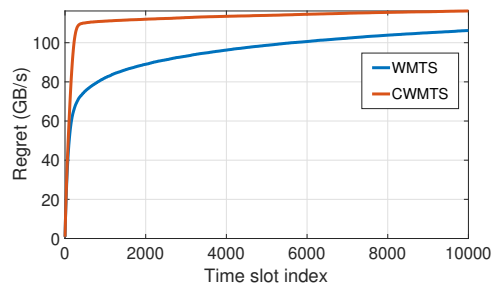

(b)

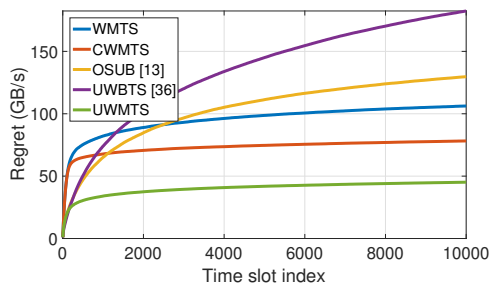

(c)

Figure 5: Regret performance based on real-world measurements: (a). No knowledge of problem structure. (b). With problem structure that instantaneous data rates are nondecreasing. (c). With problem structure that effective data rates are unimodal.

initially modeled and derived the trade-off caused by beamwidth in a multi-user mmWave network. Similar optimizations that balance the beamforming gain and the beam training overhead were also investigated in $[14,24,27]$. However, their solutions heavily depend on the physical layer assumptions or prior knowledge such as channel model, beam pattern model, and network topology, which restricts their flexibility in practical deployments in MANETs where the channel is rapidly changing. In contrast with these prior work, our proposed MAB-based solutions are model-free, and thus do not rely on the assumption of channel or user mobility.

(2) Data-driven codebook construction: Some recent work has used offline data-driven machine learning methodologies to perform beam alignment and beamwidth selection simultaneously. In [38], a deep learning technique was exploited to learn an optimal set of beam pairs by considering the environment information as feature spaces. Similarly, in [12], a large amount of experimental data were gathered to build a beamforming codebook of minimum size and subject to a guaranteed gain. Besides, a geo-located context database was built in [15] to assist the beam width/directions selection. [12, 15, 38] all showed that significant system improvement was achieved over conventional beam alignment strategies. These offline data-driven approaches however require a large amount of historical data for a given deployment site, which limits its fast implementation. Further, since they only focused on the successful connection probability of the eventually learned codebook, the trade-off between beam alignment quality and data transmission efficiency was not exploited therein. Finally, no theoretical guarantee of performance was provided.

(3) Beam alignment (including hierarchical search): Other than codebook optimization, much of the prior work focuses on selecting the best beam from a single codebook without considering the effect of beam resolution, for example, [23] proposed AgileLink which finds the best beam by a random hashing and voting mechanism. Some work exploits a priori knowledge of the channel to avoid exhausted beam search [15, 18, 29, 35, 42]. However, prior information would require additional sensors or statistics. Moreover, adaptive approaches were also investigated: $A C O$ was proposed in [30] to estimate the full channel, whereas four probes per antenna element are required, which results in poor scalability. Another approach - hierarchical search - starts (in each time slot) from a coarse beam and progressively uses finer beams to shorten the training time [28, 39]. However, it has several drawbacks: limited coverage due to the initial use of wide beams [28]; zooming in wrong directions due to beam imperfectness and interference [23]; and large feedback overhead (per measurement) in asymmetric links where devices have to respond by directional beams due to power limitation [32]. A learning-based hierarchical beam alignment strategy was proposed in [11], which sequentially learns a beamforming vector for single-path channel models, and provides notable gains in the low SNR region. In contrast, we have focused on the mmWave codebook selection by dynamically learning a sitespecific or device-specific codebook over time. Indeed, the above algorithms could be incorporated into our framework by regarding different algorithms (or an algorithm with different parameters) as different "abstract codebooks".

(4) Related bandit algorithms Thompson Sampling (TS) is a widely used method for solving MAB problems. In [25], a regret bound was shown for TS with Bernoulli arms. In [20], the weighted binary TS was derived based on [25] to deal with the case where the reward of each Bernoulli arm was multiplied by a different constant. One of the most related work is [33], in which the authors provided the regret bound for TS with multinomial arms of the same support. The above algorithms, however, do not exploit structure across arms and satisfy asymptotic optimality for unstructured bandit problems. In [21], the constrained weighted binary TS was proposed to allow incorporating general structural properties among arms. An improved performance was achieved, but an efficient implementation is still lacking (see also Section 4.3.1). To exploit reward unimodality, the OSUB algorithm was proposed in [13] based on KL-UCB. A very recent work [36] derived a theoretical guarantee for UTS with Bernoulli arms. Our proposed algorithms augment these prior studies. We highlight that we provide the first theoretical guarantees for UTS with weighted multinomial rewards.

\section{CONCLUSIONS}

In this work, we have considered the codebook selection problem in mmWave MANETs with rapidly-varying wireless channels. We have modeled it as a MAB problem and have proposed novel TS-based algorithms with/without knowing the structures among codebooks. We have derived the theoretical regret upper bounds for the proposed algorithms. The real-world mmWave measurements based evaluation has validated the benefits of our algorithms.

\section{ACKNOWLEDGMENTS}

This work was partially supported by the U.S. Army Research Office under grant W911NF1910221, NSF grants CNS-1731658 and CNS-1910112 and the US DoT supported D-STOP Tier 1 University 
Transportation Center. We thank Kartik Patel for collecting the 60 $\mathrm{GHz}$ channel traces, that was used in the simulation studies.

\section{REFERENCES}

[1] 2009. IEEE Standard for Information technology- Local and metropolitan area networks- Specific requirements- Part 15.3: Amendment 2: Millimeter-wavebased Alternative Physical Layer Extension. IEEE Std 802.15.3c-2009 (Amendment to IEEE Std 802.15.3-2003) (Oct. 2009), 1-200.

[2] 2014. WP5: Propagation, Antenna, and Multi-Antenna Techniques: D5.1 - Channe Modeling and Characterization. Technical Report. EU and Japanese Government.

[3] 2016. IEEE Standard for Information technology-Telecommunications and in formation exchange between systems Local and metropolitan area networksSpecific requirements - Part 11: Wireless LAN Medium Access Control (MAC) and Physical Layer (PHY) Specifications. IEEE Std 802.11-2016 (Revision of IEEE Std 802.11-2012) (Dec. 2016), 1-3534.

[4] 2019. IEEE Draft Standard for Information Technology-Telecommunications and Information Exchange Between Systems Local and Metropolitan Area NetworksSpecific Requirements Part 11: Wireless LAN Medium Access Control (MAC) and Physical Layer (PHY) Specifications-Amendment: Enhanced Throughput for Operation in License-Exempt Bands Above $45 \mathrm{GHz}$. IEEE P802.11ay/D4.0, fune 2019 (Jul. 2019), 1-791.

[5] 2019. System Architecture for the 5G System. document TS 23.501 V16.1.0, 3GPP, Jun. 2019 (Jun. 2019), 1-219.

[6] Shipra Agrawal and Navin Goyal. 2012. Analysis of Thompson Sampling for the Multi-armed Bandit Problem. In Proc. of the 25th Annual Conference on Learning Theory (COLT'12). Edinburgh, Scotland, 39.1-39.26.

[7] Shipra Agrawal and Navin Goyal. 2013. Further Optimal Regret Bounds for Thompson Sampling. In Proc. of the Sixteenth International Conference on Artificial Intelligence and Statistics (AISTATS'13). Scottsdale, Arizona, USA, 99-107.

[8] Peter Auer, Nicolò Cesa-Bianchi, and Paul Fischer. 2002. Finite-Time Analysis of the Multiarmed Bandit Problem. Machine Learning 47, 2-3 (May 2002), 235-256.

[9] Irmak Aykin, Berk Akgun, Mingjie Feng, and Marwan Krunz. 2020. MAMBA: A Multi-armed Bandit Framework for Beam Tracking in Millimeter-wave Systems. In Proc. of 2020 IEEE International Conference on Computer Communications (INFOCOM 2020). Shanghai, China, 1469-1478.

[10] Olivier Chapelle and Lihong Li. 2011. An Empirical Evaluation of Thompson Sampling. In Proc. of the 24th International Conference on Neural Information Processing Systems (NeurIPS'11). Granada, Spain, 2249-2257.

[11] Sung-En Chiu, Nancy Ronquillo, and Tara Javidi. 2019. Active Learning and CSI Acquisition for mmWave Initial Alignment. IEEE Journal on Selected Areas in Com munications 37, 11 (2019), 2474-2489. https://doi.org/10.1109/JSAC.2019.2933967

[12] Mohaned Chraiti, Dmitry Chizhik, Jinfeng Du, Reinaldo A. Valenzuela, Al Ghrayeb, and Chadi Assi. 2019. Beamforming Learning for mmWave Communication: Theory and Experimental Validation. arXiv ePrint 1912.12406.

[13] Richard Combes and Alexandre Proutiere. 2014. Unimodal Bandits: Regret Lowe Bounds and Optimal Algorithms. In Proc. of the 31st International Conference on Machine Learning (ICML '14). Beijing, China, 521-529.

[14] Jiancun Fan, Liyuan Han, Xinmin Luo, Ying Zhang, and Jingon Joung. 2020. Beamwidth Design for Beam Scanning in Millimeter-Wave Cellular Networks. IEEE Transactions on Vehicular Technology 69, 1 (Jan. 2020), 1111-1116.

[15] Ilario Filippini, Vincenzo Sciancalepore, Francesco Devoti, and Antonio Capone. 2018. Fast Cell Discovery in Mm-Wave 5G Networks With Context Information. IEEE Transactions on Mobile Computing 17, 7 (Jul. 2018), 1538-1552.

[16] Aurélien Garivier and Olivier Cappé. 2011. The KL-UCB Algorithm for Bounded Stochastic Bandits and Beyond. In Proc. of the 24th Annual Conference on Learning Theory (COLT'11). Budapest, Hungary, 359-376.

[17] Yasaman Ghasempour, Muhammad K. Haider, Carlos Cordeiro, Dimitrios Koutsonikolas, and Edward Knightly. 2018. Multi-Stream Beam-Training for MmWave MIMO Networks. In Proc. of the 24th Annual International Conference on Mobile Computing and Networking (MobiCom '18). New Delhi, India, 225-239.

[18] Nuria González-Prelcic, Anum Ali, Vutha Va, and Robert W. Heath. 2017. Millimeter-Wave Communication with Out-of-Band Information. IEEE Commun Mag. 55, 12 (Dec. 2017), 140-146.

[19] Aditya Gopalan, Shie Mannor, and Yishay Mansour. 2014. Thompson Sampling for Complex Online Problems. In Proc. of the 31st International Conference on on Machine Learning (ICML '14). Beijing, China, 100-108.

[20] Harsh Gupta, Atilla Eryilmaz, and R. Srikant. 2018. Low-Complexity, LowRegret Link Rate Selection in Rapidly-Varying Wireless Channels. In Proc. of 2018 IEEE International Conference on Computer Communications (INFOCOM 2018). Honolulu, HI, USA, 540-548.

[21] Harsh Gupta, Atilla Eryilmaz, and R. Srikant. 2019. Link Rate Selection using Constrained Thompson Sampling. In Proc. of 2019 IEEE International Conference on Computer Communications (INFOCOM 2019). Paris, France, 739-747.

[22] Morteza Hashemi, Ashutosh Sabharwal, C. Emre Koksal, and Ness B. Shroff. 2018. Efficient Beam Alignment in Millimeter Wave Systems Using Contextual Bandits. In Proc. of 2018 IEEE International Conference on Computer Communications
(INFOCOM 2018). Honolulu, HI, USA, 2393-2401.

[23] Haitham Hassanieh, Omid Abari, Michael Rodriguez, Mohammed Abdelghany, Dina Katabi, and Piotr Indyk. 2018. Fast Millimeter Wave Beam Alignment. In Proc. of the 2018 Conference of the ACM Special Interest Group on Data Communication (SIGCOMM '18). Budapest, Hungary, 432-445.

[24] Kishor Chandra Joshi, Solmaz Niknam, R. Venkatesha Prasad, and Balasubramaniam Natarajan. 2020. Analyzing the Tradeoffs in Using Millimeter Wave Directional Links for High Data-Rate Tactile Internet Applications. IEEE Transactions on Industrial Informatics 16, 3 (Mar. 2020), 1924-1932.

[25] Emilie Kaufmann, Nathaniel Korda, and Rémi Munos. 2012. Thompson Sampling: An Asymptotically Optimal Finite-Time Analysis. In Proc. of the 23rd International Conference on Algorithmic Learning Theory (ALT '12). Lyon, France, 199-213.

[26] Oteri Kome, Lin Cen, Lou Hanqing, and Yang Rui. 2016. Further Details on Multi-Stage, Multi-Resolution Beamforming Training in 802.11ay, doc.: IEEE 802.11-16/1447r1. Retrieved Dec. 12, 2020 from https://mentor.ieee.org/802.11/dcn/16/11-16-1447-01-00ay-further-details-onmulti-stage-multi-resolution-beamforming-training-in-802-11ay.pptx

[27] Jia Liu and Elizabeth S. Bentley. 2019. Hybrid-Beamforming-Based MillimeterWave Cellular Network Optimization. IEEE fournal on Selected Areas in Communications 37, 12 (Dec. 2019), 2799-2813.

[28] Giordani Marco, Mezzavilla Marco, and Zorzi Michele. 2016. Initial Access in 5G mmWave Cellular Networks. IEEE Commun. Mag. 54, 11 (Nov. 2016), 40-47.

[29] Thomas Nitsche, Adriana B. Flores, Edward W. Knightly, and Joerg Widmer. 2015. Steering With Eyes Closed: Mm-Wave Beam Steering Without In-Band Measurement. In Proc. of 2015 IEEE International Conference on Computer Communications (INFOCOM 2015). Kowloon, Hong Kong, China, 2416-2424.

[30] Joan Palacios, Daniel Steinmetzer, Adrian Loch, Matthias Hollick, and Joerg Widmer. 2018. Adaptive Codebook Optimization for Beam Training on Off-theShelf IEEE 802.11Ad Devices. In Proc. of the 24th Annual International Conference on Mobile Computing and Networking (MobiCom '18). New Delhi, India, 241-255.

[31] Stefano Paladino, Francesco Trovò, Marcello Restelli, and Nicola Gatti. 2017. Unimodal Thompson Sampling for Graph-Structured Arms. In Proc. of the ThirtyFirst AAAI Conference on Artificial Intelligence (AAAI '17). San Francisco, CA, USA, 2457-2463.

[32] Zhou Pei, Cheng Kaijun, Han Xiao, Fang Xuming, Fang Yuguang, He Rong, Long Yan, and Liu Yanping. 2018. IEEE 802.11ay-Based mmWave WLANs: Design Challenges and Solutions. IEEE Communications Surveys \& Tutorials 20, 3 (Thirdquarter 2018), 1654-1681.

[33] Charles Riou and Junya Honda. 2020. Bandit Algorithms Based on Thompson Sampling for Bounded Reward Distributions. In Proc. of the 31st International Conference on Algorithmic Learning Theory (ALT'20), Vol. 117. San Diego, CA, USA, 777-826.

[34] Hossein Shokri-Ghadikolaei, Lazaros Gkatzikis, and Carlo Fischione. 2015. Beamsearching and Transmission Scheduling in Millimeter Wave Communications. In 2015 IEEE International Conference on Communications. London, UK, 1292-1297.

[35] Gek Hong Sim, Sabrina Klos, Arash Asadi, Anja Klein, and Matthias Hollick. 2018. An Online Context-Aware Machine Learning Algorithm for $5 \mathrm{G}$ mmWave Vehicular Communications. IEEE/ACM Transactions on Networking 26, 6 (Dec. 2018), 2487-2500

[36] Cindy Trinh, Emilie Kaufmann, Claire Vernade, and Richard Combes. 2020. Solving Bernoulli Rank-One Bandits with Unimodal Thompson Sampling. In Proc. of the 31st International Conference on Algorithmic Learning Theory (ALT'20), Vol. 117. San Diego, CA, USA, 862-889.

[37] Song Wang, Jingqi Huang, and Xinyu Zhang. 2020. Demystifying MillimeterWave V2X: Towards Robust and Efficient Directional Connectivity under High Mobility. In Proc. of the 26th Annual International Conference on Mobile Computing and Networking (MobiCom '20). London, United Kingdom, Article 51, 14 pages.

[38] Yuyang Wang, Aldebaro Klautau, Monica Ribero, Anthony C. K. Soong, and Robert. W. Heath. 2019. MmWave Vehicular Beam Selection With Situational Awareness Using Machine Learning. IEEE Access 7 (2019), 87479-87493.

[39] Wen Wu, Nan Cheng, Ning Zhang, Peng Yang, Weihua Zhuang, and Xuemin Shen. 2019. Fast mmwave Beam Alignment via Correlated Bandit Learning. IEEE Transactions on Wireless Communications 18, 12 (Dec. 2019), 5894-5908.

[40] Zhenyu Xiao, Pengfei Xia, and Xiang-Gen Xia. 2017. Codebook Design for Millimeter-Wave Channel Estimation With Hybrid Precoding Structure. IEEE Transactions on Wireless Communications 16, 1 (Jan. 2017), 141-153.

[41] Yi Zhang, Soumya Basu, Sanjay Shakkottai, and Robert W. Heath Jr. 2021. MmWave Codebook Selection in Rapidly-Varying Channels via Multinomial Thompson Sampling. Technical Report. The University of Texas at Austin.

[42] Yi Zhang, Kartik Patel, Sanjay Shakkottai, and Robert W. Heath Jr. 2019. SideInformation-Aided Noncoherent Beam Alignment Design for Millimeter Wave Systems. In Proc. of the 20th ACM International Symposium on Mobile Ad Hoc Networking and Computing (MobiHoc '19). Catania, Italy, 341-350.

[43] Renjie Zhao, Timothy Woodford, Teng Wei, Kun Qian, and Xinyu Zhang. 2020. M-Cube: A Millimeter-Wave Massive MIMO Software Radio. In Proc. of the 26th Annual International Conference on Mobile Computing and Networking (MobiCom '20). London, United Kingdom, Article 15, 14 pages. 